\title{
Altered functional connectivity of the subthalamus and the bed nucleus of the stria terminalis in obsessive-compulsive disorder
}

\author{
M. Cano ${ }^{1,2,3}$, P. Alonso ${ }^{1,2,3}$, I. Martínez-Zalacaín ${ }^{1}$, M. Subirà ${ }^{1,3}$, E. Real ${ }^{1,3}$, C. Segalàs ${ }^{1,3}$, J. Pujol ${ }^{3,4}$, \\ N. Cardoner ${ }^{3,5,6}$, J. M. Menchón ${ }^{1,2,3}$ and C. Soriano-Mas ${ }^{1,3,7 *}$ \\ ${ }^{1}$ Department of Psychiatry, Bellvitge University Hospital-IDIBELL, L'Hospitalet de Llobregat, Barcelona, Spain \\ ${ }^{2}$ Department of Clinical Sciences, School of Medicine, University of Barcelona, Barcelona, Spain \\ ${ }^{3}$ CIBERSAM, Carlos III Health Institute, Madrid, Spain \\ ${ }^{4}$ MRI Research Unit, Radiology Department, Hospital del Mar, Barcelona, Spain \\ ${ }^{5}$ Mental Health Department, Parc Tauli Sabadell, I3PT, Barcelona, Spain \\ ${ }^{6}$ Department of Psychiatry and Legal Medicine, Universitat Autònoma de Barcelona, Barcelona, Spain \\ ${ }^{7}$ Department of Psychobiology and Methodology in Health Sciences, Universitat Autònoma de Barcelona, Barcelona, Spain
}

Background. The assessment of inter-regional functional connectivity (FC) has allowed for the description of the putative mechanism of action of treatments such as deep brain stimulation (DBS) of the nucleus accumbens in patients with obsessive-compulsive disorder (OCD). Nevertheless, the possible FC alterations of other clinically-effective DBS targets have not been explored. Here we evaluated the FC patterns of the subthalamic nucleus (STN) and the bed nucleus of the stria terminalis (BNST) in patients with OCD, as well as their association with symptom severity.

Methods. Eighty-six patients with OCD and 104 healthy participants were recruited. A resting-state image was acquired for each participant and a seed-based analysis focused on our two regions of interest was performed using statistical parametric mapping software (SPM8). Between-group differences in FC patterns were assessed with two-sample $t$ test models, while the association between symptom severity and FC patterns was assessed with multiple regression analyses.

Results. In comparison with controls, patients with OCD showed: (1) increased FC between the left STN and the right premotor cortex, (2) decreased FC between the right STN and the lenticular nuclei, and (3) increased FC between the left BNST and the right frontopolar cortex. Multiple regression analyses revealed a negative association between clinical severity and FC between the right STN and lenticular nucleus.

Conclusions. This study provides a neurobiological framework to understand the mechanism of action of DBS on the STN and the BNST, which seems to involve brain circuits related with motor response inhibition and anxiety control, respectively.

Received 3 November 2016; Revised 12 July 2017; Accepted 12 July 2017; First published online 22 August 2017

Key words: Functional connectivity, obsessive-compulsive disorder, subthalamic nucleus and bed nucleus of the stria terminalis.

\section{Introduction}

Obsessive-compulsive disorder (OCD) is a disabling disorder that affects $2-3 \%$ of the general population (Kessler et al. 2012). Despite advances in effective pharmacological and behavioural treatments, an estimated $10 \%$ of patients with OCD remain treatmentresistant and continue to suffer from severe symptoms (Denys, 2006). Deep brain stimulation (DBS) has been proposed as an alternative treatment for this group of patients with treatment-resistant OCD.

* Address for correspondence: C. Soriano-Mas, Ph.D., Department of Psychiatry, Bellvitge University Hospital, Bellvitge Biomedical Research Institute (IDIBELL), Feixa Llarga s/n, 08907, L'Hospitalet de Llobregat, Barcelona, Spain.

(Email: csoriano@idibell.cat)
DBS consists of the implantation of electrodes that send electrical pulses to deep brain areas. Since dysfunction in cortico-striato-thalamo-cortical (CSTC) circuits is central to most of the prevailing neurobiological models of OCD (Menzies et al. 2008; Milad \& Rauch, 2012; Eng et al. 2015; van den Heuvel et al. 2016), structures of the basal ganglia such as the ventral striatum and the subthalamic nucleus (STN) have become the main anatomical targets of DBS (Alonso et al. 2015).

Research on the brain functional changes induced by DBS has shown some convergence with neuroimaging studies describing functional abnormalities in OCD patients. Figee et al. (2013) showed that DBS targeted at the nucleus accumbens (NA) reduced functional connectivity (FC) of this region with the prefrontal cortex, while different studies have consistently reported 
increased FC between these structures in OCD (Harrison et al. 2009; 2013; Jung et al. 2013). Such concurring findings provide a neurobiological framework to understand the mechanism of action of DBS in OCD.

There is a lack, however, of similar studies focusing on the other basal ganglia targets of DBS for OCD. For example, no studies to date have specifically assessed the FC of the STN in patients with OCD. The STN plays a key role in indirect and hyperdirect CSTC pathways (Jahanshahi et al. 2015), and abnormalities in its FC may therefore critically account for the suspected imbalance between different CSTC pathways in OCD (Graybiel \& Rauch, 2000; Mataix-Cols \& van den Heuvel, 2006; van den Heuvel et al. 2010).

Moreover, clinical research has started to investigate other DBS targets beyond the CSTC pathways for patients with treatment-resistant OCD. Among them, the bed nucleus of the stria terminalis (BNST) stands out as one of the most targeted regions (Neumann et al. 2014; Islam et al. 2015; Luyten et al. 2016). The BNST has classically been associated with sustained anxiety responses (Walker et al. 2009; Somerville et al. 2010; Alvarez et al. 2011), and it is considered part of the extended amygdala because of its location and its strong structural and functional interactions with this medial temporal lobe structure (Davis et al. 2010). In this context, despite there being no reports of altered BNST function, FC of the amygdala seems to be altered in OCD, with reports of reduced FC at rest (Göttlich et al. 2014), task-related increases of FC with frontal regions (de Vries et al. 2014), and associations with cognitive-behavioural therapy response (Göttlich et al. 2015). Therefore, an assessment of FC of the BNST is warranted to provide an explanatory framework of the alleged effectiveness of DBS targeting this structure in refractory OCD.

The aim of this study was to assess the FC of the STN and the BNST in a large sample of OCD patients in comparison with an equally large sample of healthy control subjects. Moreover, to further investigate the importance of such FC patterns for OCD pathophysiology, its association with OCD severity was also explored. Despite this being essentially an exploratory study, we hypothesized that FC of these two nuclei with structures within CSTC circuits would be altered in OCD, and that such alterations would be associated with disorder severity.

\section{Material and methods}

\section{Participants}

A total of 86 outpatients were recruited from the OCD Unit of Bellvitge University Hospital, Barcelona, Spain. Psychiatric diagnoses were established using the
Structured Clinical Interview for DSM-IV Axis I Disorders-Clinician Version (First et al. 1997). A primary diagnosis of OCD was given if OCD symptoms were persistent and constituted the primary cause of distress and interference in the patient's life. Exclusion criteria were being aged younger than 18 or older than 65 , current or history of psychotic disorders, mental retardation, any severe organic or neurological disease other than tic disorder, current or past substance abuse/dependence, presence of any contraindication to magnetic resonance imaging (MRI), or the presence of any abnormality in the MRI scan. Each patient was assessed using the Yale-Brown Obsessive-Compulsive Scale (YBOCS) (Goodman et al. 1989), the YBOCS Symptom Checklist (Goodman et al. 1989), the Hamilton Rating Scale for Anxiety (HRSA) (Hamilton, 1959) and the Hamilton Rating Scale for Depression (HRSD) (Hamilton, 1960).

The control sample included 104 healthy participants of comparable age and gender in relation to patients. In order to rule out the possibility of current or lifetime psychiatric disorders and the use of psychotropic medication, subjects from the comparison group underwent a medical anamnesis and the Structured Clinical Interview for DSM-IV Axis I Disorders nonpatient version (First et al. 2002). The rest of exclusion criteria were the same used for the OCD group. The sociodemographic characteristics of all participants and the clinical characteristics of patients with OCD are described in Table 1.

After receiving approval from the ethical committee of clinical research (CEIC) of Bellvitge University Hospital, all participants gave written informed consent to participate in this study, which was performed in accordance with the Declaration of Helsinki.

\section{Image acquisition and preprocessing}

A 1.5-T Signa Excite system (General Electric, Milwaukee, Wisconsin) equipped with an eight-channel phased-array head coil and single-shot echo-planar imaging software was used. The functional sequence consisted of gradient recalled acquisition in the steady state (repetition time, $2000 \mathrm{~ms}$; echo time, $50 \mathrm{~ms}$; and pulse angle, $90^{\circ}$ ) in a $24-\mathrm{cm}$ field of view, with a $64 \times$ 64 pixel matrix and a slice thickness of $4 \mathrm{~mm}$ (interslice gap, $1 \mathrm{~mm}$ ). Twenty-two interleaved sections, parallel to the anterior-posterior commissure line, were acquired to generate 120 whole-brain volumes, excluding four initial dummy volumes. Participants were instructed to simply relax, stay awake, and to lie still without moving, while keeping their eyes closed throughout image acquisition. We also acquired a highresolution T1-weighted anatomical image for each subject using a three-dimensional fast spoiled gradient 
Table 1. Sociodemographic and clinical characteristics of the study samples

\begin{tabular}{|c|c|c|c|c|}
\hline Sociodemographic and clinical variables & Patients with OCD $(n=86)$ & Healthy participants $(n=104)$ & Statistic $^{\mathrm{a}}$ & $p$ Value \\
\hline Age, years: mean (s.D.) & $34.38(9.39)$ & $34.18(10.40)$ & 0.139 & 0.889 \\
\hline Gender, male: $n(\%)$ & $43(50)$ & $59(56.70)$ & 0.858 & 0.354 \\
\hline Age at onset, years: mean (S.D.) & $21.50(7.91)$ & & & \\
\hline Illness duration, years: mean (S.D.) & $12.91(10.37)$ & & & \\
\hline \multicolumn{5}{|l|}{ YBOCS: mean (S.D.) } \\
\hline Obsessions & $13.20(2.81)$ & & & \\
\hline Compulsions & $13.46(2.67)$ & & & \\
\hline Total score & $26.54(5.40)$ & & & \\
\hline HRSA: mean (S.D.) & $15.81(5.68)$ & & & \\
\hline HRSD: mean (S.D.) & $12.81(4.60)$ & & & \\
\hline \multicolumn{5}{|l|}{ Obsessive-compulsive symptoms: $n(\%)$} \\
\hline Aggressive/checking & $67(77.90)$ & & & \\
\hline Sexual/religious & $25(29.06)$ & & & \\
\hline Symmetry/ordering & $40(46.51)$ & & & \\
\hline Contamination/cleaning & $38(44.18)$ & & & \\
\hline Hoarding & $26(30.23)$ & & & \\
\hline Tic disorder: $n(\%)$ & $10(11.63)$ & & & \\
\hline \multicolumn{5}{|l|}{ Pharmacological treatment: $n(\%)$} \\
\hline No treatment & $3(3.49)$ & & & \\
\hline SSRIs & $28(32.56)$ & & & \\
\hline Clomipramine & $8(9.30)$ & & & \\
\hline Antipsychotic potentiation & $22(25.59)$ & & & \\
\hline SSRIs + Clomipramine & $24(27.90)$ & & & \\
\hline MAOI & 1 (1.16) & & & \\
\hline
\end{tabular}

OCD, obsessive-compulsive disorder; YBOCS, Yale-Brown obsessive-compulsive scale; HRSA, Hamilton Rating Scale for anxiety; HRSD, Hamilton Rating Scale for depression; SSRIs, selective serotonin reuptake inhibitors; MAOI, monoamine oxidase inhibitor.

${ }^{\text {a }}$ Independent samples $t$ test for continuous variables, $\chi^{2}$ test for categorical variables.

inversion-recovery prepared sequence with 130 contiguous slices (repetition time, $11.8 \mathrm{~ms}$; echo time, $4.2 \mathrm{~ms}$; flip angle, $15^{\circ}$ ) in a $30-\mathrm{cm}$ field of view, with a $256 \times$ 256 pixel matrix and a slice thickness of $1.2 \mathrm{~mm}$.

Imaging data were processed on a Microsoft Windows platform using technical computing software (MATLAB 7.14; The MathWorksInc, Natick, Mass) and Statistical Parametric Mapping (SPM8; The Welcome Department of Imaging Neuroscience, London, UK). After an initial pre-alignment step to the first image of the time-series, motion correction was performed by aligning (within subject) each timeseries to the mean image using a least-squares minimisation and a 6-parameter (rigid body) spatial transformation. These realigned functional sequences were subsequently coregistered to the each participant's anatomical scan, which had been previously coregistered and normalized to the SPM-T1 template. Normalization parameters were then applied to the coregistered functional images, which were smoothed with an $8 \mathrm{~mm}$ full-width at half-maximum (FWHM) isotropic Gaussian kernel.

\section{Regions of interest}

We extracted the signal from four seed-regions of interest (two per hemisphere), centred on the bed nucleus of the stria terminalis (BNST) region and the subthalamic nucleus (STN) region. Seeds were defined with the MarsBar region-of-interest toolbox as $2 \mathrm{~mm}$ radial spheres centred at the following MNI coordinates (following Höflich et al. 2013 and Krüger et al. 2015): (i) BNST region $[x= \pm 5, y=0, z=4]$ and (ii) STN region $[x= \pm 10.3, y=-16.7, z=-1]$. All these four seeds were spatially separated between each other by at least 8 mm (>1 FWHM), according to the formula:

$\sqrt{ }\left(\left(x_{1}-x_{2}\right)^{2}+\left(y_{1}-y_{2}\right)^{2}+\left(z_{1}-z_{2}\right)^{2}\right)$

where $\left(x_{1} y_{1} z_{1} \& x_{2} y_{2} z_{2}\right)$ refer to the coordinates of any two voxels in MNI space, which allowed us to obtain specific FC maps for each region. For anatomical reference, online Supplementary Fig. S1 depicts the location of our seeds of interest overlaid on normalized structural images from a selected group of study participants. 
In addition to our signals of interest, we derived estimates of white matter, cerebrospinal fluid (CSF) and global brain signal fluctuations to be included as nuisance variables in first-level analyses. Specifically, white matter and CSF SPM tissue probability maps were eroded so to keep voxels with a probability of at least 0.2 or 0.6 of being white matter or CSF, respectively. Such tissue-specific masks were then binarized to create nuisance variable masks, together with a binary mask for global brain signal, which was the sum of the white matter and CSF masks plus a gray matter mask. Across the time-series, nuisance signals were derived from each mask by averaging signal from all in-mask voxels.

\section{Statistical analyses}

Sociodemographic data were compared between groups using the Statistical Package for Social Sciences (SPSS) v.21 (SPSS Inc., Chicago, IL).

Regarding imaging analyses, first-level FC maps of each seed were calculated for each participant by estimating the regression coefficient between the seed's and each brain voxel's time series using an SPM multiple regression model. A high-pass filter set at $128 \mathrm{sec}-$ onds was used to remove low-frequency drifts of less than approximately $0.008 \mathrm{~Hz}$, and, before model estimation, the three nuisance covariates were mutually orthogonalized using an iterative Gram-Schmidt procedure. First-level FC images of each participant were then included in a second-level (group) analysis. We used an independent two-sample model to derive $t$-statistic maps comparing the FC patterns between patients with OCD and healthy participants. Specifically, we estimated 4 SPM models, resulting from the analysis of the four seeds of interest (left BNST, right BNST, left STN and right STN regions). In each model, we initially estimated positive and negative FC patterns of each group, which were thresholded at a significance threshold of $p<0.05$, Family-Wise error (FWE) corrected for multiple comparisons across the whole brain. These group specific patterns were then combined to create a mask in which we investigated between-group differences in FC.

In addition, we assessed the association between clinical severity and FC patterns using multiple regression analyses. More specifically, clinical severity, as measured by YBOCS score, was included as an independent predictor in the SPM multiple regression models to evaluate its relationship with BNST and STN FC patterns. Importantly, this analysis was focused on the pattern of significant results derived from the above between-group comparisons. In all between-group comparisons and regression analyses, statistical significance was set at $p<0.05$, FWE corrected for multiple comparisons across all in-mask voxels (i.e. using small-volume correction procedures across all voxels showing positive or negative FC in patients or controls with our seed regions of interest).

Likewise, to assess for the association between other sociodemographic and clinical variables and FC alterations, we conducted a series of analyses with the FC estimates from the peak coordinates of the above analyses. Thus, we assessed Pearson correlations with age, age at onset, illness duration, HRSA and HRSD scores and obsessive-compulsive symptom dimension scores. Also, to evaluate the potential effects of pharmacological treatment on our findings, we performed two kinds of two-sample $t$ test comparisons. Firstly, we compared those patients receiving no pharmacological or standard treatments (i.e. SSRIs or Clomipramine) $v$. those on other regimens denoting higher pharmacological resistance (i.e. antipsychotic augmentation, SSRIs + Clomipramine or MAO inhibitors, see Table 1). Secondly, we compared patients with $v$. those without dopaminergic medications. Finally, a two-sample $t$ test comparison was also used to evaluate potential between-sex differences. In these analyses, which were performed in SPSS v.21 (SPSS Inc., Chicago, IL), we used a statistical significance threshold of $p<0.05$ after a Bonferroni correction for multiple comparisons.

Finally, in a complementary analysis, to assure our analyses were based on the FC patterns of our regions of interest and that we were not capturing signal from surrounding structures, online Supplementary Figs S2 and S3 depict the patterns of functional connectivity from our seeds of interest as compared with the patterns of surrounding structures (nucleus accumbens and substantia nigra pars compacta).

\section{Results}

\section{Sociodemographic and clinical characteristics}

As can be seen in Table 1, groups did not differ in sociodemographic characteristics. Table 1 also presents the descriptive statistics of the clinical variables from the OCD group.

\section{Neuroimaging analyses}

\section{Between-group comparisons}

In comparison with healthy participants, patients with OCD showed: (1) increased FC between the left STN and the right pre-motor cortex (rPMC); (2) decreased FC between the right STN and the bilateral lenticular nuclei (bLN), including the left putamen and the right globus pallidus (rGP); and (3) increased FC between the left BNST and the right frontopolar cortex (rFPC). The FC pattern of the right BNST did 
Table 2. Brain areas showing functional connectivity differences between patients with OCD and healthy participants

\begin{tabular}{|c|c|c|c|c|c|c|c|}
\hline Seed region & $X$ & $Y$ & $z$ & $t$ value & $p$ Value $^{\mathrm{a}}$ & & Peak location \\
\hline ISTN & 26 & -26 & 56 & 4.55 & 0.034 & & rPMC (121 voxels) \\
\hline \multirow[t]{2}{*}{ rSTN } & -22 & 12 & -8 & 5.19 & 0.004 & bLN (6108 voxels) & 1Putamen \\
\hline & 18 & 6 & 2 & 5.16 & 0.004 & & rGP \\
\hline IBNST & 2 & 60 & -6 & 6.07 & $<0.001$ & & rFPC (790 voxels) \\
\hline
\end{tabular}

ISTN, left subthalamic nucleus; rSTN, right subthalamic nucleus; 1BNST, left bed nucleus of the stria terminalis; rPMC, right pre-motor cortex; bLN, bilateral lenticular nuclei; lputamen, left putamen; rGP, right globus pallidus; rFPC, right frontopolar cortex. $x, y, z$-coordinates are reported in standard Montreal Neurological Institute (MNI) space.

${ }^{\text {a }}$ FWE corrected for multiple comparisons.

not significantly differ between groups (see Table 2 and Fig. 1).

For replication of previous findings involving alterations in FC of the ventral striatum, we also evaluated between-group differences in the FC patterns of the nucleus accumbens. These results are presented in online Supplementary Fig. S4.

\section{Relationship with clinical severity and other clinical and sociodemographic factors}

The multiple regression analysis using clinical severity (YBOCS score) as the independent predictor of the FC patterns revealed a significant $(t=2.22 ; p=0.044)$ negative association between clinical severity and the FC estimate between the rSTN and the right LN ( $x=20$, $y=6, z=2$ ) (see Fig. 2). No further correlations with clinical severity were observed. Likewise, post-hoc analyses did not reveal any significant relationship between the other clinical and sociodemographic variables (including age, sex, age at onset, illness duration, HRSA and HRSD scores, symptom subtypes and pharmacological regimen) and FC alterations.

Finally, we performed an exploratory analysis to evaluate the possible association between the FC patterns of our regions of interest and the age and sex of our control subjects. These results of these analyses, reported in online Supplementary Fig. S5, showed increased FC between both BNST and right thalamus in women.

\section{Discussion}

In the present study we investigated potential alterations in the FC of two different DBS targets used for patients with refractory OCD: the STN and the BNST. We observed: (1) increased FC between the left STN and the right PMC, (2) decreased FC between the right STN and the bilateral LN, including the left putamen and the right GP, and (3) increased FC between the left BNST and the right FPC. In addition, the FC between the right STN and the right LN was negatively associated with clinical severity. These findings provide a neurobiological framework to interpret future results on the neurobiological mechanisms accounting for the effectiveness of DBS on the STN and the BNST as a treatment for OCD.

The STN is crucially involved in response inhibition capacity (see Chambers et al. 2009; Aron, 2011 or Bari \& Robbins, 2013 for a review). Specifically, as a central relay structure of the hyperdirect and the indirect CSTC pathways, it receives direct excitatory input from the prefrontal cortex (i.e. inferior frontal cortex and pre-supplementary motor area (pre-SMA)) and inhibitory input from the external globus pallidus, respectively. In turn, it provides excitatory output to the internal globus pallidus, which results in a net inhibition of thalamo-cortical activity and consequent inhibited motor response (Nambu et al. 2002; Jahanshahi et al. 2015; Rae et al. 2015). The results reported here show that, while FC with cortical structures providing input to the STN is not altered in OCD, FC with basal ganglia structures (e.g. globus pallidus) may be decreased, which could account for the decreased inhibition of motor responses that has been consistently reported in OCD. Indeed, we also observed increased connectivity between the STN and rPMC, which in all likelihood stems from disruptions in motor inhibitory pathways. Thus, according to the CSTC circuits model depicted in Fig. $3 a$, in healthy conditions it is expected that STN activity will be coupled with decreased activity in motor and premotor areas, the last step of central motor programming before sending excitatory inputs to the spinal cord (Nambu et al. 2002; Rae et al. 2015). Conversely, we report increased connectivity between these two regions, which could imply that patients with OCD may show decreased inhibitory response because of decreased FC between the STN and the internal part of the GP, resulting in a net excitation of the motor cortices.

Hyperactivity of premotor and motor cortices and decreased activation in the basal ganglia, including the caudate, the putamen and the GP, has been consistently 

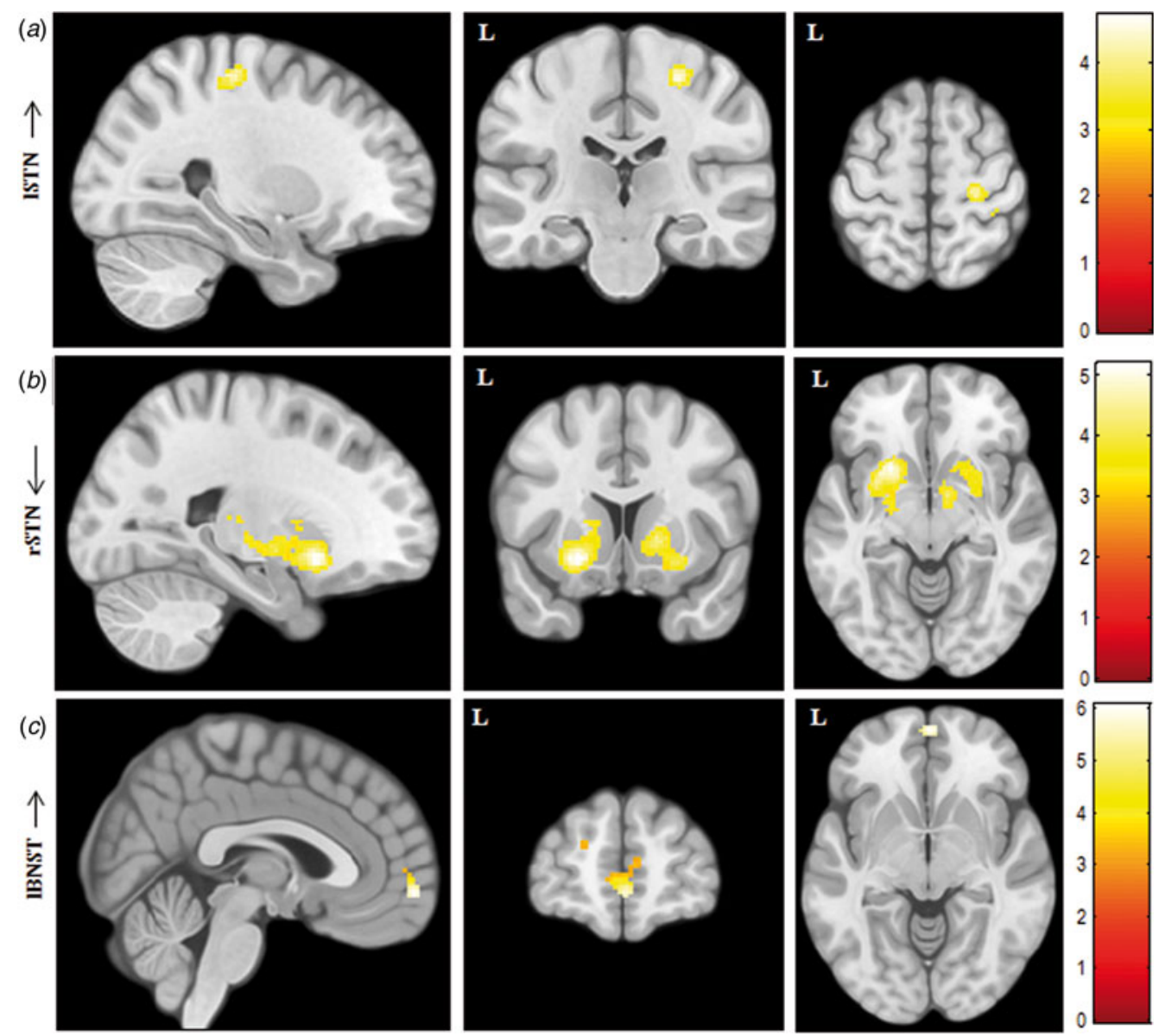

Fig. 1. (a) Functional connectivity between left subthalamic nucleus (STN) and the right pre-motor cortex, increased in patients with obsessive-compulsive disorder (OCD). (b) Functional connectivity between the right subthalamic nucleus and the bilateral lenticular nuclei, decreased in patients with OCD. (c) Functional connectivity between the left bed nucleus of the stria terminalis (BNST) and right frontopolar cortex, increased in patients with OCD. Color bar represents $t$ values. $\mathrm{L}$ indicates left hemisphere. Voxels are thresholded at $p<0.001$ (uncorrected) for illustrative purposes.

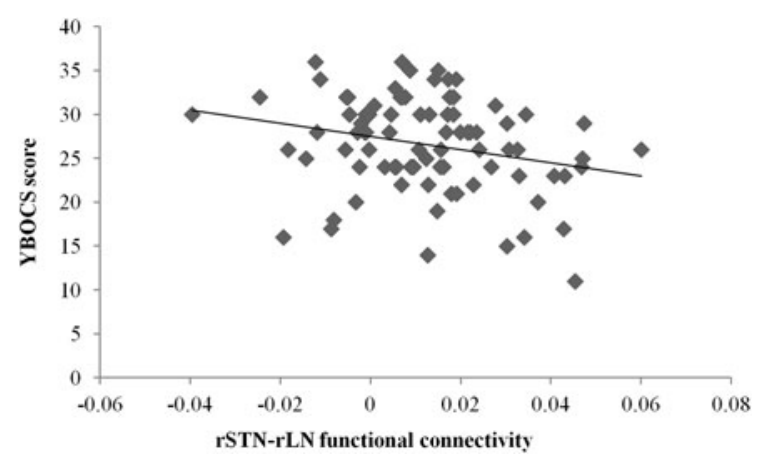

Fig. 2. Scatter plot depicting the association between right subthalamic-right lenticular nuclei (rSTN-rLN) functional connectivity and clinical severity (YBOCS score).

reported in OCD, especially during motor inhibition tasks (see Van Velzen et al. 2014 for review). Indeed, patients with OCD show increased stop-signal reaction time and higher error rates on go/no-go paradigms compared with healthy controls (Chamberlain \& Sahakian, 2007; Penadés et al. 2007; Kang et al. 2013, but also see Kalanthroff et al. 2016). Such alterations in motor inhibition have been proposed as a behavioural endophenotype of the disorder (Chamberlain \& Sahakian, 2007; Menzies et al. 2007).

Likewise, we also observed that decreased FC between the STN and the LN was associated with disorder severity. These findings were restricted to the right hemisphere, and such greater relative relevance of right CSTC circuits is in agreement with the right lateralization of motor inhibitory pathways in healthy controls (Aron \& Poldrack, 2006). Nevertheless, at more lenient significance thresholds, the left STN also showed FC alterations with bilateral lenticular nuclei (data not shown). Therefore, our results are also consistent with the 'efficient-inhibition hypothesis', which stresses the role of the left hemisphere for efficient response inhibition (Hirose et al. 2012). However, since the significant correlation with disease 


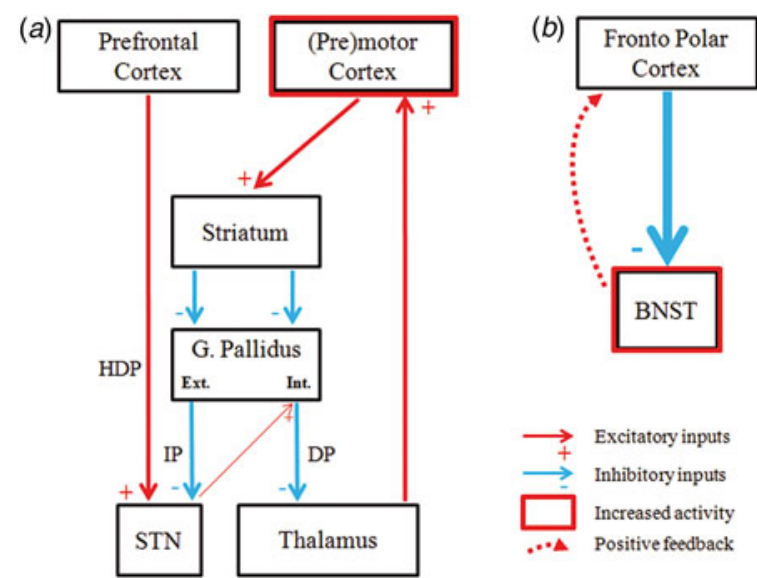

Fig. 3. Schematic representation of our findings and tentative explanatory hypotheses. (a). According to our data, within the cortico-striatal-thalamo-cortical circuits, patients with OCD showed reduced functional connectivity between the subthalamic nucleus (STN) and the internal globus pallidus, which leads to a disinhibition of the (pre)motor cortex. HDP, hyperdirect pathway; IP, indirect Pathway; DP, direct pathway. (b). The fronto polar cortex showed increased functional connectivity with the bed nucleus of the stria terminalis (BNST), likely as a consequence of the increased excitatory feedback received from this structure. In $a$ and $b$, thinner and thicker lines refer to alterations in functional connectivity in OCD patients.

severity was restricted to right hemisphere structures, our results may be supportive of the intriguing hypotheses that right unilateral DBS of the STN might be a reasonable treatment alternative. Indeed, unilateral DBS of the STN has been shown to modulate motor and cognitive symptoms in Parkinson's disease (Hershey et al. 2008). Likewise, in OCD, unilateral treatments have already been explored for other targets, such as the nucleus accumbens, showing partial clinical response (Huff et al. 2010).

Regarding our findings of increased FC between the BNST and the FPC in patients with OCD, this should be interpreted in the context of anxiety processing. It is well accepted that, although OCD is not classified as an anxiety disorder in the latest version of the Diagnostic and Statistical Manual of Mental Disorders (American Psychiatric Association, 2013), it shares many behavioural and neural characteristics with other anxiety disorders (van den Heuvel et al. 2016). Indeed, anxiety and distress are important modulators of OCD symptomatology (Gillan et al. 2016), and limbic circuits involved in anxiety processing have been typically included in neurobiological models of OCD (Milad \& Rauch, 2012). In this context, it should be noted that the BNST has been considered as part of the extended amygdala (Davis et al. 2010), although, whereas the amygdala has been related with transient anxious responses, the BNST has been associated with more sustained anxious responses (Davis et al. 2010; Somerville et al. 2010; Alvarez et al. 2011).

Activity in the FPC, as in other medial prefrontal structures, has been shown to downregulate hyperactivation of subcortical limbic structures (Etkin et al. 2009). Indeed, increased FC between the FPC and the amygdala has been observed during threat-induced anxiety (Gold et al. 2015), and similar findings could be expected regarding FPC-BNST connectivity during sustained anxiety, although this has not been explored. In any case, the FPC is indeed functionally connected with the BNST (Torrisi et al. 2015). Therefore, our findings may provide a tentative explanation regarding increased anxiety responses in OCD. We hypothesize that OCD anxiety symptoms may be related with BNST hyperactivation, and that increased FC with the FPC may result from the (unsuccessful) attempts of this cortical region to downregulate abnormally increased BNST activation (see Fig. 3b), though the correlational nature of the study precludes firm conclusions.

This study has a number of limitations. Firstly, most patients were on medication. Therefore, we cannot determine what effect, if any, concurrent pharmacological treatment had on our results. However, all patients had been stably medicated for at least 3 months before undergoing image acquisition. Moreover, we observed no differences between patients on standard pharmacological regimens and those on treatments denoting a certain degree of pharmacological resistance or with dopaminergic drugs. In any case, it is important to bear in mind that the present study was motivated by the use of the two structures assessed as DBS targets in OCD, and patients undergoing DBS have generally been medicated for a long time. Therefore, the population assessed here is representative of this group of patients with OCD. Secondly, we did not assess possible FC alterations of other clinically effective DBS targets such as, for example, the inferior thalamic peduncle. In this sense, further research is needed to provide a comprehensive description of the alterations in FC associated with the different DBS targets used for OCD. Finally, our analyses have been performed with $1.5 \mathrm{~T}$ data, which hinders the localization of tiny subcortical structures such as the ones assessed here. Further research with ultra-high field neuroimaging (i.e. $7 \mathrm{~T}$ ), as recently reported by Gorka et al. 2017, will certainly permit a better localization of these structures.

In conclusion, this study provides the first evidence of altered FC in patients with OCD from two subcortical regions that are increasingly being used as DBS targets for refractory OCD. The STN showed decreased FC with the lenticular nucleus (including the putamen 
and the globus pallidum) and increased FC with the premotor cortex, which may relate with the impaired motor response inhibition observed in OCD populations. Likewise, the BNST showed greater FC with the frontopolar cortex, most likely as a consequence of the increased basal tone of this subcortical structure and the attempts of the prefrontal cortex to downregulate its activity and therefore control anxiety symptoms. Such results provide a neurobiological framework to understand the mechanism of action of DBS treatment for OCD.

\section{Supplementary material}

The supplementary material for this article can be found at https://doi.org/10.1017/S0033291717002288

\section{Acknowledgements}

This study was supported in part by the Carlos III Health Institute (PI13/01958, PI13/00918, PI14/00413 and CIBER-CB06/03/0034); FEDER funds ('A way to build Europe') and by the Agency of University and Research Funding Management of the Catalan Government (2014SGR1672). M.C. is supported by a grant from the Spanish Ministry for Education, Culture and Sport (FPU13/02141). M.S. is supported by a Río Hortega contract (CM15/00189), E.R. by a Juan Rodés contract (JR14/00038), and C.S.-M. by a Miguel Servet contract (CPII16/00048) from the Carlos III Health Institute.

\section{Declaration of Interest}

None.

\section{References}

Alonso P, Cuadras D, Gabriëls L, Denys D, Goodman W, Greenberg BD, Jimenez-Ponce F, Kuhn J, Lenartz D, Mallet L, Nuttin B, Real E, Segalas C, Schuurman R, du Montcel ST, Menchon JM (2015). Deep brain stimulation for obsessive-compulsive disorder: a meta-analysis of treatment outcome and predictors of response. PLOS ONE 10, e0133591.

Alvarez RP, Chen G, Bodurka J, Kaplan R, Grillon C (2011). Phasic and sustained fear in humans elicits distinct pattern of brain activity. NeuroImage 55, 389-400.

American Psychiatric Association (2013). Diagnostic and Statistical Manual of Mental Disorders, 5th edn. American Psychiatric Association: Arlington, VA, USA.

Aron AR (2011). From reactive to proactive and selective control: developing a richer model for stopping inappropriate responses. Biological Psychiatry 69, 55-68.
Aron AR, Poldrack RA (2006). Cortical and subcortical contributions to stop signal response inhibition: role of the subthalamic nucleus. Journal of Neuroscience 26, 2424-2433.

Bari A, Robbins TW (2013). Inhibition and impulsivity: behavioral and neural basis of response inhibition. Progress in Neurobiology 108, 44-79.

Chamberlain SR, Sahakian BJ (2007). The neuropsychiatry of impulsivity. Current Opinion in Psychiatry 20, 255-261.

Chambers CD, Garavan H, Bellgrove MA (2009). Insights into the neural basis of response inhibition from cognitive and clinical neuroscience. Neuroscience and Biobehavioral Reviews 33, 631-646.

Davis M, Walker DL, Miles L, Grillon C (2010). Phasic vs sustained fear in rats and humans: role of the extended amygdale in fear vs anxiety. Neuropsychopharmacology 35, 105-135.

de Vries FE, de Wit SJ, Cath DC, van der Werf YD, van der Borden V, van Rossum TB, van Balkom AJ, van der Wee NJ, Veltman DJ, van den Heuvel OA (2014).

Compensatory frontoparietal activity during working memory: an endophenotype of obsessive-compulsive disorder. Biological Psychiatry 76, 878-887.

Denys D (2006). Pharmacotherapy of obsessive-compulsive disorder and obsessive-compulsive spectrum disorders. Psychiatric Clinics of North America 29, 553-584.

Eng GK, Sim K, Chen SH (2015). Meta-analytic investigations of structural grey matter, executive domain-related functional activations, and white matter diffusivity in obsessive compulsive disorder: an integrative review. Neuroscience and Biobehavioral Reviews 52, 233-257.

Etkin A, Prater KE, Schatzberg AF, Menon V, Greicius MD (2009). Disrupted amygdalar subregion functional connectivity and evidence of a compensatory network in generalized anxiety disorder. Archives of General Psychiatry 66, 1361-1372.

Figee $M$, Luigjes J, Smolders R, Valencia-Alfonso CE, van Wingen G, de Kwaasteniet B, Mantione M, Ooms P, de Koning P, Vulink N, Levar N, Droge L, van den Munckhof P, Schuurman PR, Nederveen A, van den Brink W, Mazaheri A, Vink M, Denys D (2013). Deep brain stimulation restores frontostriatal network activity in obsessive-compulsive disorder. Nature Neuroscience 16, 386-387.

First MB, Spitzer RL, Gibbon M, Williams JBW (1997). Structured Clinical Interview for DSM-IV Axis I Disorders Clinician Version (SCID-CV). American Psychiatric Press, Inc.: Washington, DC, USA.

First MB, Spitzer RL, Gibbon M, Williams JBW (2002). Structured Clinical Interview for DSM-IV Axis I Disorders Non-Patient Edition (SCID-I/NP). Biometrics Research, New York State Psychiatric Institute: New York.

Gillan CM, Robbins TW, Sahakian BJ, van den Heuvel OA, van Wingen G (2016). The role of habit in compulsivity. European Neuropsychopharmacology 26, 828-840.

Gold AL, Morey RA, McCarthy G (2015). Amygdala-prefrontal cortex functional connectivity during threat-induced anxiety and goal distraction. Biological Psychiatry 77, 394-403.

Goodman WK, Price LH, Rasmussen SA, Mazure C, Fleischmann RL, Hill CL, Heninger GR, Charney DS 
(1989). The yale-brown obsessive compulsive scale I. Development, use, and reliability. Archives of General Psychiatry 46, 1006-1011.

Gorka AX, Torrisi S, Shackman AJ, Grillon C, Ernst M (2017). Intrinsic functional connectivity of the central nucleus of the amygdala and bed nucleus of the stria terminalis. Neuroimage. http://dx.doi.org/10.1016/j. neuroimage.2017.03.007.

Göttlich M, Krämer UM, Kordon A, Hohagen F, Zurowski B (2014). Decreased limbic and increased fronto-parietal connectivity in unmedicated patients with obsessivecompulsive disorder. Human Brain Mapping 35, 5617-5632.

Göttlich M, Krämer UM, Kordon A, Hohagen F, Zurowski B (2015). Resting-state connectivity of the amygdala predicts response to cognitive behavioral therapy in obsessive compulsive disorder. Biological Psychology 111, 100-109.

Graybiel AM, Rauch SL (2000). Toward a neurobiology of obsessive-compulsive disorder. Neuron 28, 343-347.

Hamilton M (1959). The assessment of anxiety states by rating. British Journal of Medical Psychology 32, 50-55.

Hamilton M (1960). A rating scale for depression. Journal of Neurology, Neurosurgery and Psychiatry 23, 56-62.

Harrison BJ, Pujol J, Cardoner N, Deus J, Alonso P, López-Solà $\mathrm{M}$, Contreras-Rodríguez $\mathrm{O}$, Real $\mathrm{E}$, Segalàs $\mathrm{C}$, Blanco-Hinojo L, Menchon JM, Soriano-Mas C (2013). Brain corticostriatal systems and the major clinical symptom dimensions of obsessive-compulsive disorder. Biological Psychiatry 73, 321-328.

Harrison BJ, Soriano-Mas C, Pujol J, Ortiz H, López-Solà M, Hernández-Ribas R, Deus J, Alonso P, Yücel M, Pantelis C, Menchon JM, Caronder N (2009). Altered corticostriatal functional connectivity in obsessive-compulsive disorder. Archives of General Psychiatry 66, 1189-1200.

Hershey T, Wu J, Weaver PM, Perantie DC, Karimi M, Tabbal SD, Perlmutter JS (2008). Unilateral vs. Bilateral STN DBS effects on working memory and motor function in Parkinson disease. Experimental Neurology 210, 402-408.

Hirose S, Chikazoe J, Watanabe T, Jimura K, Kunimatsu A, Abe O, Ohtomo K, Miyashita Y, Konishi S (2012). Efficiency of go/no go task performance implemented in the left hemisphere. Journal of Neuroscience 32, 9059-9065.

Höflich A, Savli M, Comasco E, Moser U, Novak K, Kasper S, Lanzenberger R (2013). Neuropsychiatric deep brain stimulation for translational neuroimaging. Neurolmage 79, $30-41$.

Huff W, Lenartz D, Schormann M, Lee SH, Kuhn J, Koulousakis A, Mai J, Daumann J, Maarouf M, Klosterkötter J, Sturm V (2010). Unilateral deep brain stimulation of the nucleus accumbens in patients with treatment-resistant obsessive-compulsive disorder: outcomes after one year. Clinical Neurology and Neurosurgery 112, 137-143.

Islam L, Franzini A, Messina G, Scarone S, Gambini O (2015). Deep brain stimulation of the nucleus accumbens and bed nucleus of stria terminalis for obsessive-compulsive disorder: a case series. World Neurosurgery 83, 657-663.

Jahanshahi M, Obeso I, Rothwell JC, Obeso JA (2015). A fronto-striato-subthalamic-pallidal network for goal-directed and habitual inhibition. Nature Reviews Neuroscience 16, 719-732.

Jung WH, Kang DH, Kim E, Shin KS, Jang JH, Kwon JS (2013). Abnormal corticostriatal-limbic functional connectivity in obsessive-compulsive disorder during reward processing and resting-state. NeuroImage: Clinical $\mathbf{2 8}$ 27-38.

Kalanthroff E, Teichert T, Wheaton MG, Kimeldorf MB, Linkovski O, Ahmari SE, Fyer AJ, Schneier FR, Anholt GE, Simpson HB (2016). The role of response inhibition in medicated and unmedicated obsessive-compulsive disorder patients: evidence from the stop-signal task. Depression and Anxiety 34, 301-306.

Kang DH, Jang JH, Han JY, Kim JH, Jung WH, Choi JS, Choi CH, Kwon JS (2013). Neural correlates of altered response inhibition and dysfunctional connectivity at rest in obsessive-compulsive disorder. Progress in Neuro-Psychopharmacology and Biological Psychiatry 10, 340-346.

Kessler RC, Petukhova M, Sampson NA, Zaslavsky AM, Wittchen H-U (2012). Twelve-month and lifetime prevalence and lifetime morbid risk of anxiety and mood disorders in the United States. International Journal of Methods in Psychiatric Research 21, 169-184.

Krüger O, Shiozawa T, Kreifelts B, Scheffler K, Ethofer T (2015). Three distinct fiber pathways of the bed nucleus of the stria terminalis to the amygdale and prefrontal cortex. Cortex 66, 60-68.

Luyten L, Hendrickx S, Raymaekers S, Gabriëls L, Nuttin B (2016). Electrical stimulation in the bed nucleus of the stria terminalis alleviates severe obsessive-compulsive disorder. Molecular Psychiatry 21, 1272-1280.

Mataix-Cols D, van den Heuvel OA (2006). Common and distinct neural correlates of obsessive-compulsive and related disorders. Psychiatric Clinics of North America 29, 391-410.

Menzies L, Achard S, Chamberlain SR, Fineberg N, Chen $\mathrm{CH}$, del Campo N, Sahakian BJ, Robbins TW, Bullmore E (2007). Neurocognitive endophenotypes of obsessive-compulsive disorder. Brain 130, 3223-3236.

Menzies L, Chamberlain SR, Laird AR, Thelen SM, Sahakian BJ, Bullmore ET (2008). Integrating evidence form neuroimaging and neuropsychological studies of obsessive-compulsive disorder: the orbitofrontal-striatal model revisited. Neuroscience and Biobehavioral Reviews 32, 525-549.

Milad MR, Rauch SL (2012). Obsessive-compulsive disorder: beyond segregated cortico-striatal pathways. Trends in Cognitive Sciences 16, 43-51.

Nambu A, Tokuno H, Takada M (2002). Functional significance of the cortico-subthalamo-pallidal 'hyperdirect' pathway. Neuroscience Research 43, 111-117.

Neumann WJ, Huebl J, Brücke C, Gabriëls L, Bajbouj M, Merkl A, Schneider GH, Nuttin B, Brown P, Kühn AA (2014). Different patterns of local field potentials from limbic DBS targets in patients with major depressive and obsessive compulsive disorder. Molecular Psychiatry 19, 1186-1192. 
Penadés R, Catalán R, Rubia K, Andrés S, Salamero M, Gastó C (2007). Impaired response inhibition in obsessive compulsive disorder. European Psychiatry 22, 404-410.

Rae CL, Hughes LE, Anderson MC, Rowe JB (2015). The prefrontal cortex achieves inhibitory control by facilitating subcortical motor pathway connectivity. Journal of neuroscience 35, 786-794.

Somerville LH, Whalen PJ, Kelley WM (2010). Human bed nucleus of the stria terminalis indexes hypervigilant threat monitoring. Biological Psychiatry 68, 416-424.

Torrisi S, O'Connell K, Davis A, Reynolds R, Balderston N, Fudge JL, Grillon C, Ernst M (2015). Resting state connectivity of the bed nucleus of the stria terminalis at ultra-high field. Human Brain Mapping 36, 4076-4088.

van den Heuvel OA, van der Werf YD, Verhoef KM, de Wit S, Berendse HW, Wolters ECh, Veltman DJ, Groenewegen
HJ (2010). Frontal-striatal abnormalities underlying behaviours in the compulsive-impulsive spectrum. Journal of the Neurological Sciences 289, 55-59.

van den Heuvel OA, van Wingen G, Soriano-Mas C, Alonso P, Chamberlain SR, Nakamae T, Denys D, Goudriaan AE, Veltman DJ (2016). Brain circuitry of compulsivity. European Neuropsychopharmacology 26, 810-827.

van Velzen LS, Vriend C, de Wit SJ, van den Heuvel OA (2014). Response inhibition and interference control in obsessive-compulsive spectrum disorders. Frontiers in Human Neuroscience 8, 419.

Walker DL, Miles LA, Davis M (2009). Selective participation of the bed nucleus of the stria terminalis and CRF in sustained anxiety-like versus phasic fear-like responses. Progress in Neuro-psychopharmacology and Biological Psychiatry 13, 1291-1308. 